\title{
Exact Free Energies of Statistical Systems on Random Networks
}

\author{
Naoki SASAKURA ${ }^{\dagger}$ and Yuki SATO $\ddagger$ \\ † Yukawa Institute for Theoretical Physics, Kyoto University, Kyoto 606-8502, Japan \\ E-mail: sasakura@yukawa.kyoto-u.ac.jp \\ $¥$ National Institute for Theoretical Physics, Department of Physics and Centre \\ for Theoretical Physics, University of the Witwartersrand, WITS 2050, South Africa \\ E-mail:Yuki.Sato@wits.ac.za
}

Received June 10, 2014, in final form August 07, 2014; Published online August 15, 2014

http://dx.doi.org/10.3842/SIGMA.2014.087

\begin{abstract}
Statistical systems on random networks can be formulated in terms of partition functions expressed with integrals by regarding Feynman diagrams as random networks. We consider the cases of random networks with bounded but generic degrees of vertices, and show that the free energies can be exactly evaluated in the thermodynamic limit by the Laplace method, and that the exact expressions can in principle be obtained by solving polynomial equations for mean fields. As demonstrations, we apply our method to the ferromagnetic Ising models on random networks. The free energy of the ferromagnetic Ising model on random networks with trivalent vertices is shown to exactly reproduce that of the ferromagnetic Ising model on the Bethe lattice. We also consider the cases with heterogeneity with mixtures of orders of vertices, and derive the known formula of the Curie temperature.
\end{abstract}

Key words: random networks; exact results; phase transitions; Ising model; quantum gravity

2010 Mathematics Subject Classification: 05C82; 37A60; 46N55; 82B20; 81U15; 83C15

\section{Introduction}

The Ising model is a theory of magnets with only nearest-neighbor interactions sitting on a lattice. It is a simple but tremendously useful model in physics which allows us to describe cooperative phenomena at a critical point. Its effectiveness, in fact, is not exclusive to physics, but expands to various disciplines such as chemistry, metallurgy, mathematics and biology. Therefore, needless to say, the Ising model plays a quite important role in science.

In the history of Ising model, the most influential work has been made by Onsager in 1944 [10]: the exact solution of the 2-dimensional model has been obtained; thereby the existence of the phase transition has been proven. A significance of the exact result is to provide us a clear-cut picture of the physics near the critical point. Although 70 years have gone by since Onsager's novel work, no one has yet solved the 3-dimensional Ising model exactly. Recently, however, there was a great progress in that direction based on the conformal bootstrap [6], although still it has not given us an exact result. Other examples of the exactly solvable Ising model are, for instance, those on the 2-dimensional dynamical lattice [8] and the Bethe lattice (infinite regular tree) [2]. In the former, some simplifications exist because of enhanced symmetries. In the latter, it has been argued in [7] that regular random networks asymptotically approach the Bethe lattice in the thermodynamic limit. As can be expected from this fact, some exact results have been obtained for the Ising model on the random networks $[1,3,4,5,7,9]$, which are in accord with that on the Bethe lattice.

In this letter, we discuss a general procedure to obtain exact free energies of statistical systems on random networks with bounded but generic degrees of vertices by considering Feynman 
diagrams as random networks. As demonstrations of our method, we obtain the exact free energy of the ferromagnetic Ising model on random networks with trivalent vertices, and show that it exactly agrees with that of the Ising model on the Bethe lattice. We also consider the cases of non-regular random networks with bounded but generic orders of vertices, and derive the known formula of the Curie point $[5,9]$. We would like to comment that similar ideas of using Feynman diagrams as random networks were considered in some former works $[1,7,12]$, but the current work has some differences in methods and directions of interests. For instance, since we stick to exact expressions with real integrations rather than complex ones, the roles played by mean fields and the limitation of the mean field treatment can be clearly seen.

\section{Statistical systems on random networks with trivalent vertices}

Let us start with a statistical system on random networks with trivalent vertices. It is defined by the partition function [11]

$$
Z_{n}(K, M)=\frac{A}{n !} \int_{-\infty}^{\infty} \prod_{a=1}^{N} \mathrm{~d} \phi_{a}\left(M_{b c d} \phi_{b} \phi_{c} \phi_{d}\right)^{n} e^{-\phi_{e} K_{e f}^{-1} \phi_{f}}
$$

where $K^{-1}$ is the inverse of a symmetric real matrix $K$ with positive eigenvalues, $A=\operatorname{det} K^{-\frac{1}{2}}$, $M$ is a real symmetric three-index tensor, and the repeated indices are assumed to be summed over. Through the Gaussian integration over $\phi_{a}$ 's, the partition function (1) is represented by the summation of the Feynman diagrams, which may be regarded as random networks, with $n$ trivalent vertices weighted by $M_{a b c}$ and with $3 n / 2$ edges weighted by $K_{a b}$. The generated networks contain disconnected ones in general, but this possibility can be neglected in the thermodynamic limit $n \rightarrow \infty$, since a single connected component dominates in this limit. By rescaling $\phi_{a} \rightarrow \sqrt{n} \bar{\phi}_{a}$ in (1) and applying the Laplace method to the integral, one can obtain the exact free energy in the thermodynamic limit $n \rightarrow \infty$ [11]:

$$
f(K, M)=-\lim _{\substack{n: e v e n \\ n \rightarrow \infty}} \frac{1}{\beta}\left[\frac{1}{n} \log Z_{n}(K, M)-f_{0}(n)\right]=\frac{1}{\beta}\left(\bar{\phi}_{a} K_{a b}^{-1} \bar{\phi}_{b}-\frac{1}{2} \log \left[g(\bar{\phi})^{2}\right]\right),
$$

where $\beta$ is an inverse temperature, $f_{0}(n)=\frac{1}{2} \log n+1, g(\bar{\phi})=M_{a b c} \bar{\phi}_{a} \bar{\phi}_{b} \bar{\phi}_{c}$, and $\bar{\phi}_{a}$ ought to be real and determined in such a way as to minimize (2) for given $K, M$. Here we have subtracted $f_{0}(n)$, which is divergent in the limit $n \rightarrow \infty$, since it is independent of $K$ and $M$ and hence can be regarded as the free energy of the network rather than that of the statistical system on it. In taking the limit, we have to assume $n$ to be even, since $Z_{n=\text { odd }}=0$ for trivalent vertices. We stress here that the free energy in the thermodynamic limit (2) is exact, since the corrections to the Laplace method are of order $\log [n] / n$ and vanish in the limit.

The minimization of (2) can be realized by one of the solutions to the following extremeness condition

$$
2 K_{a b}^{-1} \bar{\phi}_{b}-\frac{3 M_{a b c} \bar{\phi}_{b} \bar{\phi}_{c}}{g(\bar{\phi})}=0 .
$$

In order to analyze the condition (3), it is useful to rescale $\bar{\phi}_{a}=g(\bar{\phi}) w_{a}$. Then $(3)$ becomes

$$
2 K_{a b}^{-1} w_{b}-3 M_{a b c} w_{b} w_{c}=0,
$$

and the free energy turns out to be

$$
f(K, M)=\frac{1}{\beta}\left(\frac{3}{2}-\frac{1}{2} \log \left[\frac{3}{2}\right]+\frac{1}{2} \log \left[w_{a} K_{a b}^{-1} w_{b}\right]\right) .
$$


Thus the free energy is determined by one of the non-vanishing solutions to (4) which minimizes (5).

The ferromagnetic Ising model with spins on vertices on random networks can be realized by taking $N=2$ and the weights as

$$
K_{a b}=\exp \left[\beta J \sigma_{a} \sigma_{b}\right], \quad M_{a b c}=\exp \left[\beta H \sigma_{a}\right] \delta_{a b} \delta_{a c}
$$

where $\sigma_{1}=1, \sigma_{2}=-1$, and $H$ and $J$ are a magnetic field and a nearest-neighbour coupling, respectively. Here we are forced to consider the ferromagnetic case, $J>0$, since all the eigenvalues of $K$ must be positive for the integration (1) to be well-defined. Then (4) are given by

$$
\begin{aligned}
& 3 e^{\beta H} \sinh [2 \beta J] w_{1}^{2}-e^{\beta J} w_{1}+e^{-\beta J} w_{2}=0, \\
& 3 e^{-\beta H} \sinh [2 \beta J] w_{2}^{2}-e^{\beta J} w_{2}+e^{-\beta J} w_{1}=0 .
\end{aligned}
$$

Note that (7) and (8) are symmetric under the simultaneous flip, $H \rightarrow-H$ and $w_{1} \leftrightarrow w_{2}$. By solving (7) for $w_{2}$, and plugging it into (8), one obtains

$$
w_{1}\left[2-6 e^{2 \beta J} \cosh [\beta(H-J)] w_{1}+18 e^{2 \beta J} \sinh [2 \beta J] w_{1}^{2}-27 e^{\beta(H+J)} \sinh [2 \beta J]^{2} w_{1}^{3}\right]=0 .
$$

Since the solutions of (9) are given by $w_{1}=0$ and those to a cubic equation, there exist explicit analytic expressions for the solutions. While these solutions are too complicated to write down in the general case, the non-vanishing solutions in the case of $H=0$ have the following simple expressions:

$$
\begin{aligned}
& \text { (I) } w_{1}=w_{2}=\frac{1}{3 \cosh [\beta]}, \\
& \text { (II) } w_{1}=\frac{1 \pm \sqrt{\frac{e^{\beta}-3 e^{-\beta}}{2 \cosh [\beta]}}}{6 \sinh [\beta]}, \quad w_{2}=\frac{1 \mp \sqrt{\frac{e^{\beta}-3 e^{-\beta}}{2 \cosh [\beta]}}}{6 \sinh [\beta]},
\end{aligned}
$$

where we have set $J=1$ for simplicity. The solution (I) is real for all $\beta$. The solutions (II) are real and minimize (5) for $\beta>\beta_{c}=\frac{1}{2} \log [3]$, but are not real and therefore inappropriate for $\beta<\beta_{c}$. Thus, by putting the solutions into (5), the free energy is determined to be

for $\beta \leq \beta_{c}$,

$$
f(\beta)=\frac{3}{2 \beta}-\frac{1}{2 \beta} \log \left[\frac{3}{2}\right]-\frac{1}{2 \beta} \log \left[9 \cosh [\beta]^{3}\right],
$$

for $\beta>\beta_{c}$,

$$
f(\beta)=\frac{3}{2 \beta}-\frac{1}{2 \beta} \log \left[\frac{3}{2}\right]-\frac{1}{2 \beta} \log \left[\frac{36 \cosh [\beta] \sinh [\beta]^{3}}{e^{\beta}-2 e^{-\beta}}\right] .
$$

From these expressions of the free energy, one can derive the specific heat, $C=-2 \beta^{2} \frac{\partial}{\partial \beta} f-$ $\beta^{3} \frac{\partial^{2}}{\partial \beta^{2}} f$ :

$$
C(\beta)= \begin{cases}\frac{3 \beta^{2}}{2 \cosh [\beta]^{2}}, & \text { for } \beta<\beta_{c} \\ \frac{3 \beta^{2}(2 \sinh [2 \beta]+\cosh [2 \beta]-2)}{2 \cosh [\beta]^{2} \sinh [\beta]^{2}\left(2-e^{2 \beta}\right)^{2}}, & \text { for } \beta>\beta_{c} .\end{cases}
$$

In the case with a non-vanishing magnetic field, it is a straightforward task to obtain the perturbative solutions, $w_{1}=w_{1}^{(0)}+w_{1}^{(1)} H+w_{1}^{(2)} H^{2}+\cdots$, of $(9)$, and compute the free energy in 
perturbation of $H$. Then one can determine the magnetization $m=-\left.\frac{\partial f}{\partial H}\right|_{H=0}$ and the magnetic susceptibility $\chi=-\left.\frac{\partial^{2} f}{\partial H^{2}}\right|_{H=0}$ as follows:

$$
\begin{aligned}
& m(\beta)= \begin{cases}0, & \text { for } \beta<\beta_{c}, \\
\frac{1}{1-2 e^{-2 \beta}} \sqrt{\frac{e^{2 \beta}-3}{e^{2 \beta}+1}}, & \text { for } \beta>\beta_{c},\end{cases} \\
& \chi(\beta)= \begin{cases}\frac{2 \beta}{3 e^{-2 \beta}-1}, & \text { for } \beta<\beta_{c}, \\
\frac{4 \beta e^{2 \beta}}{\left(2-e^{2 \beta}\right)^{2}\left(e^{4 \beta}-2 e^{2 \beta}-3\right)}, & \text { for } \beta>\beta_{c} .\end{cases}
\end{aligned}
$$

The results above show that there is a second-order phase transition point at $\beta=\beta_{c}$ with mean field features; the specific heat has a jump at the point; the magnetization and the magnetic susceptibility behave around it as $m(\beta) \sim\left(\beta-\beta_{c}\right)^{1 / 2}$ and $\chi(\beta) \sim\left(\beta-\beta_{c}\right)^{-1}$, respectively $[1,3,4,5,7,9]$. In our method, the roles of mean fields are played by $\bar{\phi}_{a}$.

In fact, one can show that the system is fully equivalent to the ferromagnetic Ising model on the Bethe lattice of trivalent vertices. Let us denote $x=w_{2} / w_{1}$. Then, from (7) and (8), one can show

$$
x=\frac{e^{\beta(-J+H)}+e^{\beta(J-H)} x^{2}}{e^{\beta(J+H)}+e^{\beta(-J-H)} x^{2}} .
$$

This is the fixed point equation of the recursive relation for solving the ferromagnetic Ising model on the Bethe lattice, and has been previously derived from saddle point analysis for $H=0$ in the approaches of $[1,7]$. From (7), one can also show

$$
w_{1}=\frac{e^{-\beta(H+J)}\left(e^{2 \beta J}-x\right)}{3 \sinh [2 \beta J]} .
$$

Then, by using (10) and (11) to express $H$ and $w_{i}$ in terms of $J$ and $x$, one obtains

$$
w_{a} K_{a b}^{-1} w_{b}=\frac{4 e^{-3 \beta J}\left(z^{2}+1-z\left(x+x^{-1}\right)\right)\left(x+x^{-1}-2 z\right)}{9\left(1-z^{2}\right)^{3}},
$$

where $z=e^{-2 \beta J}$. By putting this expression into (5), one reproduces the free energy of the ferromagnetic Ising model on the Bethe lattice [2] up to const/ $\beta$, which is irrelevant to the thermodynamic properties.

The above discussions so far for the Ising model are restricted to the ferromagnetic case, $J>0$, and below let us comment on the anti-ferromagnetic case, $J<0$, in view of our framework. The restriction comes from the fact that, for $J \leq 0, K$ in (6) has a vanishing or negative eigenvalue and (1) is ill-defined. So, to change the form of $K$, let us consider a transformation, $K_{a^{\prime} b^{\prime}}^{\prime}=K_{a b} R_{a a^{\prime}}^{-1} R_{b b^{\prime}}^{-1}, M_{a^{\prime} b^{\prime} c^{\prime}}^{\prime}=R_{a^{\prime} a} R_{b^{\prime} b} R_{c^{\prime} c} M_{a b c}$, with a matrix $R$, which is generally allowed to be complex. Obviously, this transformation does not change the statistical weights of each Feynman diagram, and therefore the statistical systems before and after the transformation can be regarded identical. Indeed it is easy to explicitly find an $R$, by which $K$ and $M$ for $J<0$ in (6) are transformed to a symmetric real matrix $K^{\prime}$ with positive eigenvalues and $M^{\prime}$ which is complex for $H \neq 0$. Then the expression (1) with $K^{\prime}$ and $M^{\prime}$ is well-defined, and gives the exact free energy for the anti-ferromagnetic case. In this case, however, we cannot apply the Laplace method to obtain (2), because the integrand is complex. As a result, the system cannot be treated with mean fields. On the other hand, the integration in (1) for such a case could be computed by the saddle point method by deforming the real integration contours to complex 
ones. However, it would be a non-trivial issue to generally show the existence of such deformed complex contours satisfying the conditions validating saddle point treatment, while this would be possible for some concrete cases $[1,7]$. We would also like to comment that, by considering the tensor products of a number of $K$ and $M$ [1], the replica trick to quench random networks can also be treated in our method.

\section{Extention to random networks with bounded but generic degrees of vertices}

From now on, we will consider random networks with bounded but generic degrees of vertices. The partition function of such a generic model is defined by

$$
Z_{n}(K, M, t)=\frac{A}{n !} \int_{-\infty}^{\infty} \prod_{a=1}^{N} \mathrm{~d} \phi_{a}\left[g_{n}(\phi)\right]^{n} e^{-\phi_{b} K_{b c}^{-1} \phi_{c}},
$$

where $g_{n}(\phi)$ is a polynomial function of $\phi_{a}$ in the form

$$
g_{n}(\phi)=\sum_{k=3}^{p} n^{-\frac{k-3}{2}} t^{(k)} M_{a_{1} \ldots a_{k}}^{(k)} \phi_{a_{1}} \cdots \phi_{a_{k}} .
$$

Here $t^{(k)}$ are variables introduced for convenience, and the scalings in $n$ are needed for all the couplings $M^{(k)}$ to play roles in dynamics, i.e., without the scalings, the dynamics will be dominated by the highest order term. This will become clear in due course.

The generic random networks generated by (12) and (13) contain 3 - to $p$-valent vertices. Since the number of vertices with degree $k$ in each contribution to the partition function can be counted by the order of $t^{(k)}$, the statistical average of vertex degree can be computed by

$$
\langle k\rangle_{n}=\frac{1}{n} \sum_{k=3}^{p} k t^{(k)} \frac{\partial}{\partial t^{(k)}} \log Z_{n}(K, M, t) .
$$

As for the thermodynamic limit, the same rescaling of $\phi_{a}$ and the application of the Laplace method as before in (12) leads to that the free energy $f(K, M, t)$ is given by the same expression as (2) with

$$
g(\bar{\phi})=\sum_{k=3}^{p} t^{(k)} M_{a_{1} \ldots a_{k}}^{(k)} \bar{\phi}_{a_{1}} \cdots \bar{\phi}_{a_{k}} .
$$

Then the average of vertex degree (14) can be expressed in the thermodynamic limit as

$$
\langle k\rangle=-\beta \sum_{k=3}^{p} k t^{(k)} \frac{\partial}{\partial t^{(k)}} f(K, M, t)=\frac{1}{g(\bar{\phi})} \bar{\phi}_{a} \frac{\partial}{\partial \bar{\phi}_{a}} g(\bar{\phi}) .
$$

Similarly, the average of vertex degree square is given by

$$
\left\langle k^{2}\right\rangle=\frac{1}{g(\bar{\phi})}\left(\bar{\phi}_{a} \frac{\partial}{\partial \bar{\phi}_{a}}\right)^{2} g(\bar{\phi}) .
$$

Let us consider the ferromagnetic Ising model on the general random networks for a vanishing magnetic field $H=0$ by taking $N=2$ and

$$
K_{a b}=\exp \left[\beta J \sigma_{a} \sigma_{b}\right], \quad M_{a_{1} a_{2} \ldots a_{k}}^{(k)}=\delta_{a_{1} a_{2}} \delta_{a_{1} a_{3}} \cdots \delta_{a_{1} a_{k}} .
$$


Hereafter we set $J=1$ for simplicity. The variables $t^{(k)}$ parametrize the weights of each kind of vertices.

The extremeness condition for $\bar{\phi}_{a}$ is given by

$$
2 K_{a b}^{-1} \bar{\phi}_{b}-\frac{\bar{g}^{\prime}\left(\bar{\phi}_{a}\right)}{\bar{g}\left(\bar{\phi}_{1}\right)+\bar{g}\left(\bar{\phi}_{2}\right)}=0
$$

where we have introduced $\bar{g}(\bar{\phi})=\sum_{k=3}^{p} t^{(k)} \bar{\phi}^{k}$, and its derivative $\bar{g}^{\prime}(\bar{\phi})$ with respect to $\bar{\phi}$. In the disordered phase, the solution is symmetric under $1 \leftrightarrow 2$, i.e., $\bar{\phi}_{1}=\bar{\phi}_{2}=\bar{\phi}$. Therefore $\bar{\phi}$ must satisfy

$$
4\left(K_{11}^{-1}+K_{12}^{-1}\right) \bar{\phi}^{2}-\langle k\rangle=0,
$$

which is obtained by substituting $\bar{\phi}_{1}=\bar{\phi}_{2}=\bar{\phi}$ into (17), and using (15) and $K_{11}=K_{22}$, $K_{12}=K_{21}$. At a second-order critical point, (17) must have degenerate solutions. This requires that the derivatives of (17) for $a=1,2$,

$$
2 K_{a b}^{-1} \mathrm{~d} \bar{\phi}_{b}-\frac{\bar{g}^{\prime \prime}\left(\bar{\phi}_{a}\right) \mathrm{d} \phi_{a}}{\bar{g}\left(\bar{\phi}_{1}\right)+\bar{g}\left(\bar{\phi}_{2}\right)}+\frac{\bar{g}^{\prime}\left(\bar{\phi}_{a}\right) \bar{g}^{\prime}\left(\bar{\phi}_{b}\right) \mathrm{d} \bar{\phi}_{b}}{\left(\bar{g}\left(\bar{\phi}_{1}\right)+\bar{g}\left(\bar{\phi}_{2}\right)\right)^{2}},
$$

must degenerate at the critical point. By taking the subtraction and addition of the two derivatives for $a=1,2$, and taking into account $\bar{\phi}_{1}=\bar{\phi}_{2}=\bar{\phi},(15)$ and (16), one can find that there exist two cases of degeneracy:

$$
\begin{aligned}
& \text { (A) } 4\left(K_{11}^{-1}-K_{12}^{-1}\right) \bar{\phi}^{2}-\langle k(k-1)\rangle=0, \\
& \text { (B) } 4\left(K_{11}^{-1}+K_{12}^{-1}\right) \bar{\phi}^{2}-\langle k(k-1)\rangle+\langle k\rangle^{2}=0 .
\end{aligned}
$$

In the case (A), by using (18), one can delete $\bar{\phi}^{2}$, and obtain

$$
\beta_{c}=\frac{1}{2} \log \frac{\left\langle k^{2}\right\rangle}{\left\langle k^{2}\right\rangle-2\langle k\rangle} .
$$

This agrees with the formula for the Curie temperature obtained in the literatures $[5,9]$. On the other hand, for the case (B), we obtain

$$
\left\langle k^{2}\right\rangle-\langle k\rangle^{2}-2\langle k\rangle=0 .
$$

The degeneracy condition of the case $(\mathrm{A})$ is obtained by the subtraction of the two derivatives, and correspondingly the degeneracy is in the direction $\mathrm{d} \phi_{1}-\mathrm{d} \phi_{2}$. This direction is odd under the exchange $1 \leftrightarrow 2$, which is associated to the flip of the magnetic field, $H \rightarrow-H$, as explained in the sentence below (8). Thus the case (A) corresponds to a critical point of spontaneous magnetization. On the other hand, in the case (B), the degeneracy is in the symmetric direction $\mathrm{d} \phi_{1}+\mathrm{d} \phi_{2}$, and the corresponding critical point, if it existed, would not be related to spontaneous magnetization.

\section{Conclusions}

We summarize our results. To begin with, we have formulated a systematic general method to obtain the exact free energies in the thermodynamic limit of statistical systems on random networks with bounded but generic degrees of vertices. Then, as demonstrations, we have discussed the ferromagnetic Ising models on random networks, and have reproduced the former results in the literatures $[1,3,4,5,7,9]$. The mean field results in the former study can be 
naturally understood by the roles of $\bar{\phi}_{a}$ in the current work. The anti-ferromagnetic case has been shown to have a difficulty in the mean-field treatment. As for the future study, we can in principle put and analyze various statistical systems on random networks with heterogeneity of degrees of vertices by considering various choices of $N, K, M$. It seems also possible to extend the method to include networks with unbounded degrees of vertices by considering nonpolynomial $g_{n}(\phi)$. It would also be interesting to pursue the connection to a model of quantum gravity [11].

\section{Acknowledgements}

We would like to thank Des Johnston for some communications.

\section{References}

[1] Bachas C., de Calan C., Petropoulos P.M.S., Quenched random graphs, J. Phys. A: Math. Gen. 27 (1994), 6121-6128, hep-th/9405068.

[2] Baxter R.J., Exactly solved models in statistical mechanics, Academic Press, Inc., London, 1982.

[3] Dembo A., Montanari A., Ising models on locally tree-like graphs, Ann. Appl. Probab. 20 (2010), 565-592, arXiv:0804.4726.

[4] Dembo A., Montanari A., Sly A., Sun N., The replica symmetric solution for Potts models on $d$-regular graphs, arXiv:1207.5500.

[5] Dorogovtsev S.N., Goltsev A.V., Mendes J.F.F., Ising model on networks with an arbitrary distribution of connections, Phys. Rev. E 66 (2002), 016104, 5 pages, cond-mat/0203227.

[6] El-Showk S., Paulos M.F., Poland D., Rychkov S., Simmons-Duffin D., Vichi A., Solving the 3D Ising model with the conformal bootstrap, Phys. Rev. D 86 (2012), 025022, 17 pages, arXiv:1203.6064.

[7] Johnston D.A., Plecháč P., Equivalence of ferromagnetic spin models on trees and random graphs, J. Phys. A: Math. Gen. 31 (1998), 475-482.

[8] Kazakov V.A., Ising model on a dynamical planar random lattice: exact solution, Phys. Lett. A 119 (1986), $140-144$.

[9] Leone M., Vázquez A., Vespignani A., Zecchina R., Ferromagnetic ordering in graphs with arbitrary degree distribution, Eur. Phys. J. B 28 (2002), 191-197, cond-mat/0203416.

[10] Onsager L., Crystal statistics. I. A two-dimensional model with an order-disorder transition, Phys. Rev. 65 (1944), 117-149.

[11] Sasakura N., Sato Y., Ising model on random networks and the canonical tensor model, Progr. Theoret. Exp. Phys. 2014 (2014), 053B03, 15 pages, arXiv:1401.7806.

[12] Whittle P., Fields and flows on random graphs, in Disorder in Physical Systems, Editors G.R. Grimmett, D. Welsh, Oxford Science Publications, Oxford University Press, New York, 1990, 337-348. 